\title{
Joint Subcarrier Pairing and Power Loading in Relay Aided Cognitive Radio Networks
}

\author{
Guftaar Ahmad Sardar Sidhu ${ }^{1}$, Feifei Gao ${ }^{1,2,3}$, Wen Chen ${ }^{\dagger}$, and Wei Wang ${ }^{\ddagger}$ \\ ${ }^{1}$ School of Engineering and Sciences, Jacobs University Bremen, 28759 Germany \\ ${ }^{2}$ National Mobile Communications Research Laboratory, Southeast University, Nanjing, China \\ 3 Tsinghua National Laboratory for Information Science and Technology, Tsinghua University, China \\ $\dagger$ Department of Electronics Engineering, Shanghai Jiaotong University, China \\ $\ddagger$ Department of Electrical Engineering, South Dakota State University, United States
}

\begin{abstract}
This paper investigates the resource allocation problem in a relay-aided cognitive radio (CR) system under the orthogonal frequency division multiplexing (OFDM) transmission. Different from the conventional $\mathrm{CR}$ resource allocation problem, the relay node here is capable of performing subcarrier permutation over two hops such that the signal received over a particular subcarrier is forwarded on a different subcarrier. The objective is to maximize the throughput of the CR network subject to a limited power budget at the secondary source and relay node, as well as the interference constraints at the primary receiver. The optimization is performed under a unified framework where the power allocation at the source node, power allocation at the relay node, and subcarrier pairing at the two hops are optimized jointly. Finally, numerical examples are provided to corroborate the proposed studies.
\end{abstract}

\section{INTRODUCTION}

Cognitive radio (CR) is a well known technique to combat the spectrum scarcity problem [1], where an unlicensed secondary user (SU) shares the spectrum of a licensed primary user (PU) either opportunistically when the PU is idle, or transmits simultaneously over the same spectrum subject to a certain interference limitation [2].

On the other hand, the orthogonal frequency division multiplexing (OFDM) based relay networks have shown their great potential to overcome the inter-symbol interference (ISI) problem and enhance the transmission efficiency. Thus the combination of OFDM transmission and CR systems becomes an important technology for the future generation wireless systems [3]- [6]. The authors in [3] studied the power allocation problem in OFDM based CR networks under the overlay transmission protocol. The work [3] was further extended to the relay-aided transmission scenario in [4]. A suboptimal algorithm that alternatingly optimize the source and relay power allocation was proposed. However, the works in [3] and [4] did not consider any power limitations on the batteries of transmitting nodes. The work [5] studied the problem of

This work was supported by the open research fund of National Mobile Communications Research Laboratory, Southeast University (No. 2011D02), by the Specialized Research Fund for the Doctoral Program of Higher Education of China (No. 20110002120059), by the German Research Foundation (DFG) under Grant GA 1654/1-1, and by Higher Education Commission (HEC) Pakistan. throughput maximization in a multi-carrier CR systems under transmit power and interference power constraints for the underlay CR scheme. The extension of [5] to an amplify-andforward (AF) relay aided transmission can be found in [6] where the power allocation in OFDM based CR system is performed under a sum power constraint. However, a more natural choice should be the individual battery power constraint for each transmitting node. Moreover, subcarrier pairing at the relay terminals in OFDM based multi-hop networks is known to enhance the spectrum efficiency [7], [8]. However, the subcarrier pairing in CR networks is a non-trivial task and to the best of the authors' knowledge, has not been explored in the literature yet.

In this work, we consider an AF relay aided CR network that is operated with the OFDM scheme and underlay transmission strategy. We seek for the optimal resource allocation for the source and relay terminals of the CR user under an interference limitation of primary user, including power allocation over different subcarriers at secondary source/relay nodes and subcarrier pairing at the secondary relay station.

\section{System Model and Problem Statement}

\section{A. System Model}

We consider a relay aided CR network where a secondary source node (SSN) communicates with the secondary destination node (SDN) with the help of a secondary relay node (SRN), as shown in Fig. 1. Underlay CR transmission is adopted where the SU simultaneously shares the whole spectrum with the PU. We assume that the SSN and the SDN are located far from each other such that the direct communication link is missing [8]. On the other hand, the primary destination node (PDN) is located close enough to both the SSN and the SRN such that it experiences interference during both transmission phases. OFDM transmission with $K$ subcarriers is applied for the CR network. Unlike the previous reported works where the authors assumed that the signal received at the SRN over a particular subcarrier is forwarded to the destination over the same subcarrier, we adopt a tone permutation policy such that the signal received on subcarrier $k$ over the SSN-to-SRN link is forwarded over subcarrier $j$ 


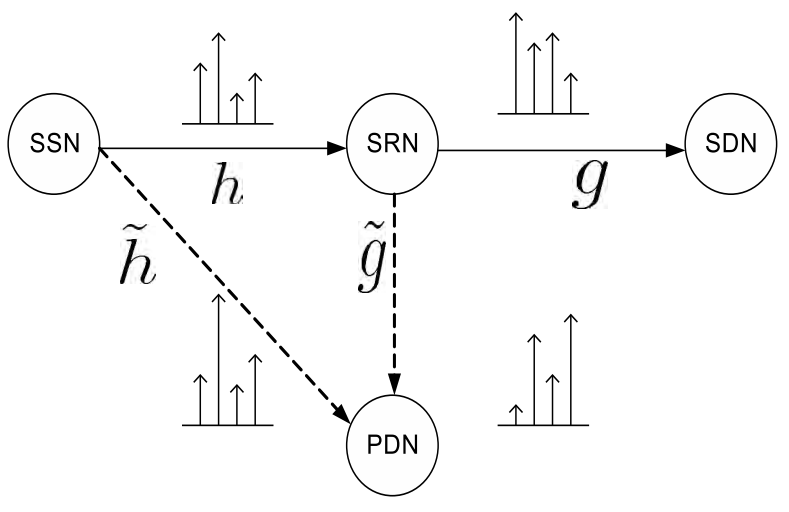

Fig. 1. Relay enhanced cognitive radio network model.

at the SRN-to-SDN link, where the index $j$ is not necessarily equal to $k$.

For the SSN to SRN, denote the channel coefficient over the $k$ th subcarrier as $h_{k}$, the power allocation as $p_{k}$, and the transmitted symbol as $x_{k}$. The signal received at the SRN over the $k$ th subcarrier is

$$
y_{k}^{\mathrm{SRN}}=\sqrt{p_{k}} h_{k} x_{k}+z_{k},
$$

where $z_{k}$ is the additive white Gaussian noise (AWGN) with variance $\sigma_{k}^{2}$. The signal received over the $j$ th subcarrier at $\mathrm{SDN}$ is then

$$
y_{j}^{S D N}=\sqrt{q_{j}} \eta_{k}\left(g_{j} h_{k} \sqrt{p_{k}} x_{k}+g_{j} z_{k}\right)+w_{j},
$$

where $\eta_{k} \triangleq\left(\sqrt{p_{k}\left|h_{k}\right|^{2}+\sigma_{k}^{2}}\right)^{-1}, q_{j}$ is the transmit power allocated to the $j$-th subcarrier, $g_{j}$ denotes the corresponding channel coefficient between the SRN and the SDN, and $w_{j}$ represents the AWGN at SDN with the variance $\sigma_{j}^{2}$.

The throughput over subcarrier pair $(k, j)$ at high signal-tonoise ratio (SNR) can be approximated by [8].

$$
c_{(k, j)} \approx \frac{1}{2} \log _{2}\left(1+\frac{\left(\sqrt{p_{k}}\left|h_{k}\right| \sqrt{q_{j}}\left|g_{j}\right|\right)^{2}}{\sigma_{j}^{2}\left(\sqrt{p_{k}}\left|h_{k}\right|\right)^{2}+\sigma_{k}^{2}\left(\sqrt{q_{j}}\left|g_{j}\right|\right)^{2}}\right),
$$

where the factor $\frac{1}{2}$ is due to the two time slots used in a complete transmission.

\section{B. Constraint Definitions}

Since each subcarrier over hop-1 can be paired with one and only one subcarrier in hop-2, we define $\pi_{(k, j)} \in\{0,1\}$ as the binary variable such that

$$
\pi_{(k, j)}=\left\{\begin{array}{l}
1, \quad \begin{array}{l}
\text { if the } k \text {-th sub-carrier of the first hop is } \\
\text { paired with the } j \text {-th sub-carrier of the } \\
\text { second hop, }
\end{array} \\
0, \quad \text { otherwise. }
\end{array}\right.
$$

With the above definition, the subcarrier pairing constraint can be mathematical expressed as

$$
\sum_{k=1}^{K} \pi_{(k, j)}=1, \forall j, \quad \sum_{j=1}^{K} \pi_{(k, j)}=1, \forall k .
$$

Under the proposed transmission protocol, the SSN and the SRN cause interference into the PDN in the first and the second time slots respectively. To protect the PDN from uncompromising interference, the power allocations at the SSN and SRN must satisfy

$$
\sum_{k=1}^{K} p_{k}\left|\tilde{h}_{k}\right|^{2} \leq I_{t h}, \quad \sum_{j=1}^{K} q_{j}\left|\tilde{g}_{j}\right|^{2} \leq I_{t h}
$$

where $\tilde{h}_{k}$ and $\tilde{g}_{j}$ denote the channel coefficients from SSN-toPDN and SRN-to-PDN links, respectively, while $I_{t h}$ denotes the maximum allowable interference threshold.

Further, we assume separate transmit power constraints at the SSN and SRN such that the summation of power allocated over all subcarriers is within the maximum available power budget of the corresponding node

$$
\sum_{k=1}^{K} p_{k} \leq P_{S S N}, \quad \sum_{j=1}^{K} q_{j} \leq P_{S R N}
$$

\section{Problem formulation}

In CR networks, a subcarrier with the highest channel gain over the first hop may also have the strongest interference to the primary users, and pairing this subcarrier with the strongest subcarrier over the second hop may result in severe performance degradation. Thus, a more considerate subcarrier pairing strategy is required that jointly considers throughput maximization of the secondary user as well as the interference mitigation to the primary user.

We jointly optimize the power allocation at the SSN, power allocation at the SRN, and subcarrier pairing at the two hops such that the overall system throughput of the secondary user is maximized subject to the maximum power constraints of the battery and the allowable interference constraints of the primary user.

Remark: The difference from the power allocation used in a conventional relay work is illustrated here. In conventional relay networks, a better power allocation scheme is to adopt $v_{i}>v_{i^{\prime}}$ when $f_{i}>f_{i^{\prime}}$, where $v_{i} \in\left\{p_{i}, q_{i}\right\}, i \in\{k, j\}$, $f_{i} \in\left\{\left|h_{i}\right|^{2},\left|g_{i}\right|^{2}\right\}$. However, in CR based relay networks, for $f_{i}>f_{i^{\prime}}$ but $\tilde{f}_{i}>\tilde{f}_{i^{\prime}}$ where $\tilde{f}_{i} \in\left\{\left|\tilde{h}_{i}\right|^{2},\left|\tilde{g}_{i}\right|^{2}\right\}$, we may need $v_{i}<v_{i^{\prime}}$.

Mathematically, we need to optimize over the variables $\boldsymbol{\pi}=$ $\left\{\pi_{(k, j)} \in\{0,1\}\right\}, \mathbf{p}=\left\{p_{k} \geq 0\right\}$ and $\mathbf{q}=\left\{q_{j} \geq 0\right\}$ for all $k=\{1, \ldots, K\}, j=\{1, \ldots, K\}$. The optimization can be formulated as

$$
\begin{aligned}
\max _{\boldsymbol{\pi}, \mathbf{p}, \mathbf{q}} & \sum_{k=1}^{K} \sum_{j=1}^{K} \pi_{(k, j)} c_{(k, j)}, \\
\text { s.t. } & (4),(5),(6) .
\end{aligned}
$$

\section{Joint Resource Allocation Scheme}

The subcarrier pairing constraint (4) makes the problem (7) a mixed integer programming and generally prohibits the duality gap to be zero. However, under the time sharing condition the duality gap is asymptotically zero for sufficiently 
large $K$ [9]. A rigorous investigation is provided in [10]. Since the time sharing condition is readily satisfied in our case, we can solve the dual problem of the original problem (7), which is defined as [11]

$$
\min _{\lambda \geq 0, \mu \geq 0, \tilde{\lambda} \geq 0, \tilde{\mu} \geq 0} D(\lambda, \mu, \tilde{\lambda}, \tilde{\mu})
$$

where $\lambda, \mu, \tilde{\lambda}, \tilde{\mu}$ are the dual variables associated with the constraints in (5) and (6), and the dual function $D(\lambda, \mu, \tilde{\lambda}, \tilde{\mu})$ is given by

$$
D(\lambda, \mu, \tilde{\lambda}, \tilde{\mu})=\max _{\mathbf{p}, \mathbf{q}, \boldsymbol{\pi}} L(\mathbf{p}, \mathbf{q}, \boldsymbol{\pi}, \lambda, \mu, \tilde{\lambda}, \tilde{\mu})
$$

s.t. (4).

The Lagrangian in (9) is defined as

$$
\begin{aligned}
& L(\mathbf{p}, \mathbf{q}, \boldsymbol{\pi}, \lambda, \mu, \tilde{\lambda}, \tilde{\mu}) \\
= & \sum_{k=1}^{K} \sum_{j=1}^{K} \pi_{(k, j)} c_{(k, j)}+\lambda\left(P_{S S N}-\sum_{k=1}^{K} p_{k}\right)+\mu\left(P_{S R N}-\right. \\
& \left.\sum_{j=1}^{K} q_{j}\right)+\tilde{\lambda}\left(I_{t h}-\sum_{k=1}^{K} p_{k}\left|\tilde{h}_{k}\right|^{2}\right)+\tilde{\mu}\left(I_{t h}-\sum_{j=1}^{K} q_{j}\left|\tilde{g}_{j}\right|^{2}\right) .
\end{aligned}
$$

\section{A. Solving the Dual Function:}

In order to solve the dual problem in (8), we need to first find the solution of (9). A Lagrange dual decomposition approach is adopted to obtain the optimum dual function for the given dual variables $\lambda, \mu, \tilde{\lambda}$, and $\tilde{\mu}$.

From the observations $\sum_{k=1}^{K} d_{k}=\sum_{k=1}^{K} \sum_{j=1}^{K} \pi_{(k, j)} d_{k}$, $\sum_{j=1}^{K} e_{j}=\sum_{k=1}^{K} \sum_{j=1}^{K} \pi_{(k, j)} e_{j}$, where $d_{k} \in\left\{p_{k}, p_{k}\left|\tilde{h}_{k}\right|^{2}\right\}$ and $e_{j} \in\left\{q_{j}, q_{j}\left|\tilde{g}_{j}\right|^{2}\right\}$, (9) can be re-stated as

$$
\begin{aligned}
& D(\lambda, \mu, \tilde{\lambda}, \tilde{\mu}) \\
= & \max _{\mathbf{p}, \mathbf{q}, \boldsymbol{\pi}} \sum_{k=1}^{K} \sum_{j=1}^{K} \pi_{(k, j)}\left(c_{(k, j)}-p_{k}\left(\lambda+\tilde{\lambda}\left|\tilde{h}_{k}\right|^{2}\right)-q_{j}(\mu+\right. \\
& \left.\left.\tilde{\mu}\left|\tilde{g}_{j}\right|^{2}\right)\right)+\lambda P_{S S N}+\mu P_{S R N}+I_{t h}(\tilde{\lambda}+\tilde{\mu}), \quad(11) \\
\text { s.t. } & \sum_{k=1}^{K} \pi_{(k, j)}=1, \forall j, \quad \sum_{j=1}^{K} \pi_{(k, j)}=1, \forall k . \\
= & \max _{\pi}\left\{\sum _ { k = 1 } ^ { K } \sum _ { j = 1 } ^ { K } \pi _ { ( k , j ) } \operatorname { m a x } _ { p _ { k } , q _ { j } } \left(c_{(k, j)}-p_{k}\left(\lambda+\tilde{\lambda}\left|\tilde{h}_{k}\right|^{2}\right)-q_{j}\right.\right. \\
& \left.\left.\left(\mu+\tilde{\mu}\left|\tilde{g}_{j}\right|^{2}\right)\right)\right\}+\lambda P_{S S N}+\mu P_{S R N}+I_{t h}(\tilde{\lambda}+\tilde{\mu}), \quad(12) \\
\text { s.t. } & \sum_{k=1}^{K} \pi_{(k, j)}=1, \forall j, \quad \sum_{j=1}^{K} \pi_{(k, j)}=1, \forall k .
\end{aligned}
$$

Thus the problem (9) is decomposed into two sub-problems.
1) Sub-problem 1: For any valid subcarrier pair $(k, j)$, i.e., $\pi_{k, j}=1$, the optimal power allocations $p_{k}^{*}$ and $q_{j}^{*}$ could be found from

$$
\begin{aligned}
\max _{p_{k}, q_{j}} & c_{(k, j)}-p_{k}\left(\lambda+\tilde{\lambda}\left|\tilde{h}_{k}\right|^{2}\right)-q_{j}\left(\mu+\tilde{\mu}\left|\tilde{g}_{j}\right|^{2}\right) \\
\text { s.t. } & p_{k} \geq 0, q_{j} \geq 0 .
\end{aligned}
$$

Since (13) is a standard convex problem, the optimal solution can be obtained from the Karush-Kuhn-Tucker (KKT) conditions as

$p_{k}^{*}=\frac{1}{\left(1+\sqrt{\frac{\alpha_{(k, j)}}{\beta_{(k, j)}}}\right)}\left(\frac{1}{\lambda+\tilde{\lambda}\left|\tilde{h}_{k}\right|^{2}}-\frac{\left(\sqrt{\alpha_{(k, j)}}+\sqrt{\beta_{(k, j)}}\right)^{2}}{a_{k} \beta_{(k, j)}}\right)^{+}$,

$q_{j}^{*}=\frac{1}{\left(1+\sqrt{\frac{\beta_{(k, j)}}{\alpha_{(k, j)}}}\right)}\left(\frac{1}{\mu+\tilde{\mu}\left|\tilde{g}_{j}\right|^{2}}-\frac{\left(\sqrt{\alpha_{(k, j)}}+\sqrt{\beta_{(k, j)}}\right)^{2}}{\alpha_{(k, j)} b_{j}}\right)^{+}$,

where $a_{k} \triangleq\left|h_{k}\right|^{2} / \sigma_{k}^{2}, b_{j} \triangleq\left|g_{j}\right|^{2} / \sigma_{j}^{2}, \alpha_{(k, j)} \triangleq a_{k}\left(\mu+\tilde{\mu}\left|\tilde{g}_{j}\right|^{2}\right)$, $\beta_{(k, j)} \triangleq\left(\lambda+\tilde{\lambda}\left|\tilde{h}_{k}\right|^{2}\right) b_{j}$, and $(v)^{+} \triangleq \max (0, v)$. Detailed derivation steps are omitted due to space limitations.

Remark: Different from the conventional water-filling solution where the power depends only on the dual variables (water-level) that are associated with the power constraint (i.e., $\lambda, \mu$ ), the water level here also depends on the dual variables that are associated with the primary user interference constraints (i.e., $\tilde{\lambda}, \tilde{\mu}$ ). Further, (14) and (15) show that the powers at the $k$-th subcarrier of the first hop and the $j$-th subcarrier of the second hop also depend on the corresponding interference channel (i.e., $\tilde{h}_{k}$ and $\tilde{g}_{j}$, respectively), resulting in a different water level for each subcarrier.

Substituting (14) and (15) into (12), the dual function becomes

$$
\begin{aligned}
& D(\lambda, \mu, \tilde{\lambda}, \tilde{\mu}) \\
& =\max _{\pi} \sum_{k=1}^{K} \sum_{j=1}^{K} \pi_{(k, j)} F_{(k, j)}(\lambda, \mu, \tilde{\lambda}, \tilde{\mu})+\lambda P_{S S N}+\mu P_{S R N} \\
& \quad+I_{t h}(\tilde{\lambda}+\tilde{\mu}), \\
& \text { s.t. } \quad \sum_{k=1}^{K} \pi_{(k, j)}=1, \forall j, \quad \sum_{j=1}^{K} \pi_{(k, j)}=1, \forall k,
\end{aligned}
$$

where the function $F_{(k, j)}$ is obtained by using the values of $p_{k}^{*}$ and $q_{j}^{*}$ in the objective of (13) and is expressed in (22)

with $x_{(k, j)} \triangleq\left(\lambda+\tilde{\lambda}\left|\tilde{h}_{k}\right|^{2}\right)\left(\sqrt{\alpha_{(k, j)}}+\sqrt{\beta_{(k, j)}}\right)^{2}$ and $y_{(k, j)} \triangleq\left(\mu+\tilde{\mu}\left|\tilde{g}_{j}\right|^{2}\right)\left(\sqrt{\alpha_{(k, j)}}+\sqrt{\beta_{(k, j)}}\right)^{2}$. Note that $F_{(k, j)}$ is independent of the power variables $p_{k}$ and $q_{j}$ and is defined for given values of the dual variables. 


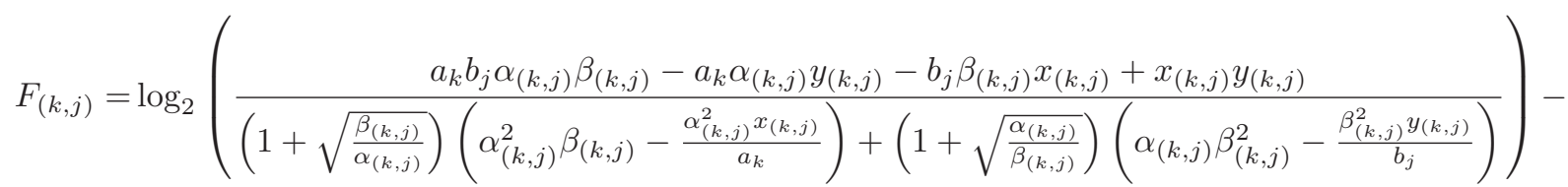

$$
\begin{aligned}
& \left(\frac{\left(a_{k} \beta_{(k, j)}-x_{(k, j)}\right)\left(b_{j} \alpha_{(k, j)}+b_{j} \sqrt{\alpha_{(k, j)} \beta_{(k, j)}}\right)+\left(b_{j} \alpha_{(k, j)}-y_{(k, j)}\right)\left(a_{k} \beta_{(k, j)}+a_{k} \sqrt{\alpha_{(k, j)} \beta_{(k, j)}}\right)}{a_{k} b_{j}\left(\beta_{(k, j)}+\sqrt{\alpha_{(k, j)} \beta_{(k, j)}}\right)\left(\alpha_{(k, j)}+\sqrt{\alpha_{(k, j)} \beta_{(k, j)}}\right)}\right) .
\end{aligned}
$$

2) Sub-problem 2: For known power allocation, the subcarrier pairing can be obtained from

$$
\max _{\pi} \sum_{k=1}^{K} \sum_{j=1}^{K} \pi_{(k, j)} F_{(k, j)}(\lambda, \mu, \tilde{\lambda}, \tilde{\mu})
$$

s.t. (4).

We define a $K \times K$ profit matrix $\boldsymbol{F}$ such that

$$
\boldsymbol{F}=\left[\begin{array}{cccc}
F_{(1,1)} & F_{(1,2)} & \ldots & F_{(1, K)} \\
F_{(2,1)} & F_{(2,2)} & \ldots & F_{(2, K)} \\
: & & : & : \\
\cdot & & . & . \\
F_{(K-1,1)} & F_{(K-1,2)} & \ldots & F_{(K-1, K)} \\
F_{(K, 1)} & F_{(K, 2)} & \ldots & F_{(K, K)}
\end{array}\right],
$$

where the row and column indices denote different customers and different items to sell, respectively, while the value of each entry can be deemed as the profit. Problem (17) is equivalent to maximizing the sum profit by an optimal selling strategy that can only sell one item to one customer. Such kind of linear assignment problem can be solved efficiently from the standard Hungarian algorithm with complexity $O\left(K^{3}\right)$ [12] and yields the optimum subcarrier pairing $\pi^{*}$.

\section{B. Solving the Dual Problem with Sub-gradient Method}

Substituting $\mathbf{p}^{*}, \mathbf{q}^{*}$ and $\pi^{*}$ into (10) and then the result back into (9), we obtain the optimal dual function for the given values of the dual variables. The optimal dual variables can be obtained from the dual problem (8) using the sub-gradient method [11]. For any initial values $\lambda^{(0)}, \mu^{(0)}, \tilde{\lambda}^{(0)}$ and $\tilde{\mu}^{(0)}$, the dual variables at the $(i+1)$-th iteration could be updated as

$$
\begin{aligned}
& \lambda^{(i+1)}=\left(\lambda^{(i)}-\delta^{(i)} \Delta^{(i)}(\lambda)\right)^{+}, \mu^{(i+1)}=\left(\mu^{(i)}-\delta^{(i)} \Delta^{(i)}(\mu)\right)^{+}, \\
& \tilde{\lambda}^{(i+1)}=\left(\tilde{\lambda}^{(i)}-\delta^{(i)} \Delta^{(i)}(\tilde{\lambda})\right)^{+}, \tilde{\mu}^{(i+1)}=\left(\tilde{\mu}^{(i)}-\delta^{(i)} \Delta^{(i)}(\tilde{\mu})\right)^{+},
\end{aligned}
$$

where $\delta^{(i)}$ is the appropriate step size of the $i$-th iteration. Moreover $\Delta(\lambda)=P_{S S N}-\sum_{k=1}^{K} p_{k}^{*}, \Delta(\mu)=P_{S R N}-$ $\sum_{j=1}^{K} q_{j}^{*}, \Delta(\tilde{\lambda})=I_{t h}-\sum_{k=1}^{K}\left|\tilde{h}_{k}\right|^{2} p_{k}^{*}$, and $\underset{\tilde{\lambda}}{\Delta}(\tilde{\mu})=I_{t h}-$ $\sum_{j=1}^{K}\left|\tilde{g}_{j}\right|^{2} q_{j}^{*}$ are the subgradients of $D(\lambda, \mu, \tilde{\lambda}, \tilde{\mu})$.

\section{Power Allocation without Subcarrier Pairing}

The computational complexity of the joint subcarrier pairing and power loading scheme in previous sub-sections increases when the number of subcarriers increases. Here we propose a suboptimal approach where optimal power allocation is obtained without considering subcarrier pairing.

Defining $\mathrm{SNR}_{k}=\frac{\left(\sqrt{p_{k}}\left|h_{k}\right| \sqrt{q_{k}}\left|g_{k}\right|\right)^{2}}{\sigma_{k}^{2}\left(\sqrt{p_{k}}\left|h_{k}\right|\right)^{2}+\sigma_{k}^{2}\left(\sqrt{q_{k}}\left|g_{k}\right|\right)^{2}}$, the optimization in (7) is then

$$
\begin{array}{cl}
\max _{\mathbf{p}, \mathbf{q}} & \sum_{k=1}^{K} \log _{2}\left(1+\mathrm{SNR}_{k}\right) \\
\text { s.t. } & (5),(6), \quad \text { with } j=k,
\end{array}
$$

which can be solved through the dual decomposition technique in similar way as in subsections III-A and III-B. The optimum power variables $p_{k}^{*}$ and $q_{k}^{*}$ could be found from (14) and (15) by replacing $j$ with $k$. Similarly, the dual variable updates are obtained from (19) and (20).

\section{Simulation Results}

This section provides numerical examples to validate the performance of the developed schemes. We assume 6-tap channels taken from i.i.d. Gaussian random variables for all links, and choose $K=32$. Without loss of generality we assume that $P_{S S N}=P_{S R N}=P_{\max }$. Further, the noise variances at SRN and SDN are set as $\sigma_{k}^{2}=\sigma_{j}^{2}, \forall k, j$. The figure of the merit is taken as the per tone rate of the secondary user, i.e., the sum rate divided by $K$. We compare three different algorithms. 1) JntSol: The joint optimization solution presented in Section III. 2) PowSol: The algorithm where only the power allocation is performed while subcarrier pairing is not considered. 3) SUB: A suboptimal algorithm based on the equal power allocation scheme developed in [5] for the single hop system, where at each node the power is equally distributed among the carriers subject to the condition that the interference constraint is satisfied. Specifically, for our system the powers are

$$
p_{k}=\min \left(\frac{P_{S S N}}{K}, \frac{I_{t h}}{\left.\sum_{k=1}^{K} \tilde{\mid} \tilde{h}_{k}\right|^{2}}\right), q_{j}=\min \left(\frac{P_{S R N}}{K}, \frac{I_{t h}}{\left.\sum_{j=1}^{K} \tilde{\mid} \tilde{g}_{j}\right|^{2}}\right) .
$$

In the first example, the performance comparison of three different algorithms are shown in Fig. 2. We display the secondary system's throughput versus $P_{\max }$ at $I_{t h}=10$. We observe that the JntSol exhibits the best performance at all $P_{\max }$ values among all different schemes. Moreover, the power optimization schemes without pairing also provide 


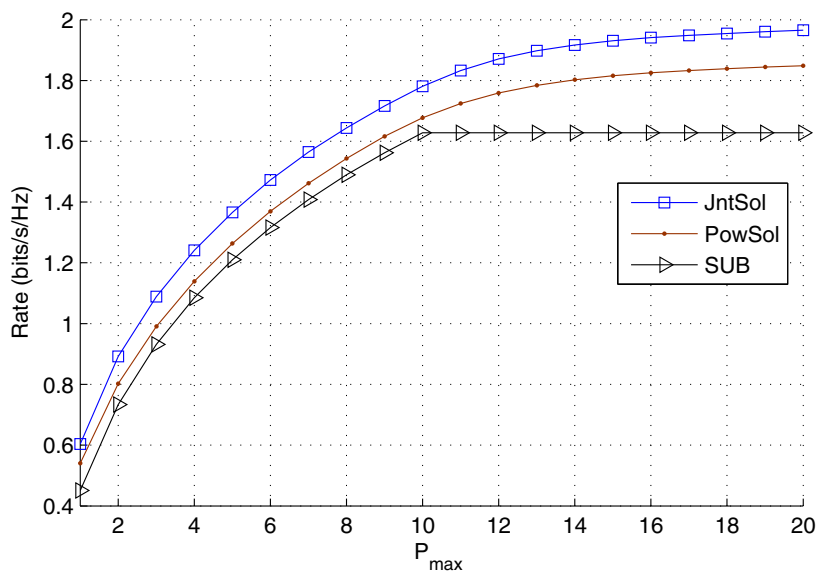

Fig. 2. Rate versus $P_{\max }$

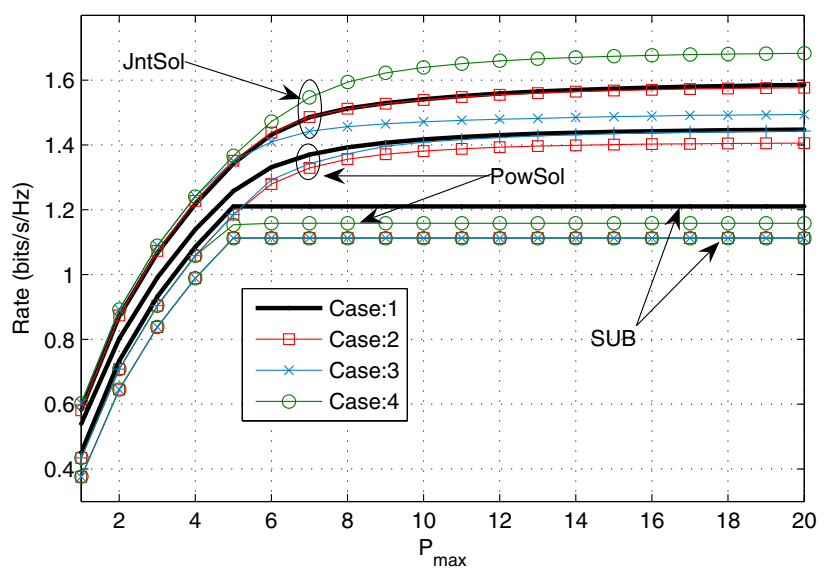

Fig. 3. Rate versus $P_{\max }$.

much better performance over the trivial method i.e., SUB algorithm.

To obtain a more insight on the impact of subcarrier pairing, in this example we compare JntSol with PowSol and SUB for the following four cases. Case-1: Same as in Fig. 2.

Case-2: $\left|h_{1}\right|^{2}>\left|h_{2}\right|^{2} \ldots>\left|h_{K}\right|^{2}$ and $\left|g_{1}\right|^{2}<\left|g_{2}\right|^{2} \ldots<$ $\left|g_{K}\right|^{2}$.

Case-3: $\left|h_{1}\right|^{2}>\left|h_{2}\right|^{2} \ldots>\left|h_{K}\right|^{2},\left|\widetilde{h}_{1}\right|^{2}>\left|\widetilde{h}_{2}\right|^{2} \ldots>\left|\widetilde{h}_{K}\right|^{2}$, $\left|g_{1}\right|^{2}<\left|g_{2}\right|^{2} \ldots<\left|g_{K}\right|^{2}$, and $\left|\widetilde{g}_{1}\right|^{2}<\left|\widetilde{g}_{2}\right|^{2} \ldots<\left|\widetilde{g}_{K}\right|^{2}$.

Case-4: $\left|h_{1}\right|^{2}>\left|h_{2}\right|^{2} \ldots>\left|h_{K}\right|^{2},\left|\widetilde{h}_{1}\right|^{2}<\left|\widetilde{h}_{2}\right|^{2} \ldots<\left|\widetilde{h}_{K}\right|^{2}$, $\left|g_{1}\right|^{2}<\left|g_{2}\right|^{2} \ldots<\left|g_{K}\right|^{2}$, and $\left|\widetilde{g}_{1}\right|^{2}>\left|\widetilde{g}_{2}\right|^{2} \ldots>\left|\widetilde{g}_{K}\right|^{2}$.

The throughput versus $P_{\max }$ for the four cases at $I_{t h}=5$ is shown in Fig. 3. It can be seen that in Case-2 JntSol exhibits almost the same performance as in Case-1 where PowSol and SUB experience the performance loss. This is because in the PowSol and SUB algorithms the stronger carrier at first hop will always be matched to the weaker carrier over the second hop. For Case-3, all the schemes (JntSol, PowSol, SUB) undergo rate loss as compared to the Case-1. The PowSol and the SUB algorithms observe loss for a similar reason as in Case-2 while JntSol experiences performance degradation due to the fact that a subcarrier with the highest interference gain is always paired with a subcarrier of the highest interference. Nevertheless, JntSol still exhibits the best performance over the others. In Case-4, we first observe that the solution with equal power allocation is the same as in Case-2 and Case-3. However, PowSol and JntSol exhibit the most interesting behavior, i.e., PowSol experiences a significant performance degradation and JntSol yields much better performance compared to all other cases. The gain in JntSol is due to the fact that the strongest subcarrier (with the smallest interference) is paired with the strongest subcarrier (with the weakest interference) where PowSol suffers from the loss because a stronger subcarrier (with low interference) is paired to a weaker subcarrier (with higher interference).

\section{COnClusions}

In this work, we investigated the resource allocation problem in relay aided OFDM cognitive radio networks. The secondary user's throughput is maximized via joint subcarrier pairing and power allocation. Meanwhile, the interference from both the secondary source and the secondary relay node to the primary receiver is kept within an acceptable limit. A suboptimal resource allocation algorithm was also designed to reduce the computational complexity. The numerical examples demonstrated enhanced performance compared to trivial resource allocation algorithms.

\section{REFERENCES}

[1] J. Miltola and G.Q. Maguire, "Cognitive radio: making software radios more personal," IEEE Personal Commun., vol. 6, no. 6, pp. 13-18, Aug. 1999.

[2] S. Haykin, "Cognitive radio brain-empowered wireless communications," IEEE J. on Sel. Areas in Commun., vol. 23, no. 2, pp. 201-220, Feb. 2005.

[3] G. Bansal, M. J. Hossain, and V. K. Bhargava, "Adaptive power loading for OFDM-based cognitive radio systems," in Proc. IEEE Int. Conf. Commun. (ICC), pp. 5137-5142, Glasgow, Scotland, June 2007.

[4] S. Yan, and X. Wang, "Power allocation for cognitive radio systems based on nonregenerative OFDM relay transmission," in Proc. IEEE Int. Conf. on Wireless Commun., Networking and Mobile Computing (WiCom), pp. 1-4, Beiging, China, Sept. 2009.

[5] K. Son, B. C. Jung; S. Chong, and D. K. Sung, "Opportunistic underlay transmission in multi-carrier cognitive radio systems," in Proc. IEEE Int. Conf. Wireless Commun. and Networking (WCNC), pp. 1-6, Budapast, Hungary, April, 2009.

[6] M. K. Shamim, M. K. Ahmed, and R. A. Shah, "Power allocation in OFDM-based cognitive relay networks," in Proc. IEEE Int. Conf. Wireless Commun., Networking and Information Security (WCNIS), pp. 202-206, Beiging, China, June, 2010.

[7] M. Herdin, "A chunk based OFDM amplify-and-forward relaying scheme for 4G mobile radio systems," in Proc. IEEE Int. Conf. Commun. (ICC), pp. 4507-4512, Istanbul, Turkey, June 2006.

[8] I. Hammerstrom and A. Wittneben, "Power allocation schemes for amplify-and-forward MIMO-OFDM relay links, " IEEE Trans. Wireless Commun., vol. 6, pp. 2798-2802, Aug. 2007.

[9] W. Yu and R. Lui, "Dual methods for nonconvex spectrum optimization of multicarrier systems," IEEE Trans. Commun., vol. 54, no. 7, pp. 13101322, July 2006.

[10] Z. Luo and S. Zhang, "Dynamic spectrum management: complexity and duality," IEEE J. Sel. Topics Sign. Proc.,, vol. 2, no. 1, pp. 57-73, Feb. 2008.

[11] S. Boyd and L. Vandenberghe, Convex Optimization. Cambridge University Press, 2004.

[12] H. Khun, "The hungarian method for the assignment problems," Naval Research Logistics Quarterly 2, pp. 83-97, 1955. 